\title{
MiR-345-5p inhibits tumorigenesis of papillary thyroid carcinoma by targeting SETD7
}

\author{
Ming Zhao, Kejing Wang, Jinbiao Shang, Zhong Liang, Weihui Zheng, Jialei Gu
}

Department of Head and Neck Surgery, Zhejiang Cancer Hospital, Zhejiang, China

Submitted: 27 November 2018

Accepted: 8 January 2019

Arch Med Sci 2020; 16 (4): 888-897

DOI: https://doi.org/10.5114/aoms.2019.83823

Copyright @ 2019 Termedia \& Banach

\section{Abstract}

Introduction: This study aimed to explore the effects of miR-345-5p on papillary thyroid carcinoma (PTC) and uncover the possible mechanisms.

Material and methods: MiR-345-5p and SETD7 mRNA levels were analyzed by quantitative real-time PCR and SETD7 protein level was measured by Western blot. The viability, colony formation ability and apoptosis of PTC cells were measured with CCK-8, soft agar colony formation and flow cytometry assay, respectively. Luciferase reporter assay was used to identify miR-345-5p's target.

Results: Compared to neighboring normal tissues, there was lower miR345-5p expression and higher SETD7 expression in PTC tissues. Moreover, Spearman's correlation analysis indicated that there was a negative correlation between miR-345-5p and SETD7 expression in PTC tissues. MiR-345$5 p$ mimics inhibited the viability and colony formation of TPC1 and B-CPAP cells and promoted apoptosis, whereas anti-miR-345-5p promoted PTC cell proliferation and inhibited apoptosis. SETD7 was confirmed to be a direct target of miR-345-5p through target scan analysis and luciferase reporter assay. Additionally, overexpression of SETD7 promoted the viability and colony formation of TPC1 and B-CPAP cells and inhibited apoptosis, whereas downregulation of SETD7 by shRNAs had opposite effects on PTC cells. Furthermore, overexpression of SETD7 attenuated the miR-345-5p induced anti-tumor effects on PTC cells.

Conclusions: MiR-345-5p exhibited suppressive effects on PTC via targeting SETD7.

Key words: miR-345-5p, papillary thyroid carcinoma, proliferation, apoptosis, SETD7.

\section{Introduction}

A variety of risk factors are associated with the occurrence of thyroid cancer, nearly $80 \%$ of cases of which are papillary thyroid carcinoma (PTC) $[1,2]$. In recent years, PTC incidence has been steadily increasing, especially in young females [3-5]. Patients with small localized PTC have a good prognosis [6, 7]. However, nearly $10 \%$ of patients develop recurrence and metastasis within 10 years [8]. Thus, there is an urgent need for exploration of the molecular mechanisms underlying the initiation and pathogenesis of PTC in order to obtain better diagnosis and therapy of PTC.

MiRNAs are small (approximately 22 nucleotides) noncoding RNAs which regulate gene expression $[5,9,10]$. Mature miRNAs pair with the $3^{\prime}$-untranslated regions (3'-UTRs) of specific mRNAs and lead to mRNA degradation

\author{
Corresponding author: \\ Kejing Wang \\ Department of Head \\ and Neck Surgery \\ Zhejiang Cancer Hospital \\ Hangzhou \\ Zhejiang Province, China \\ Phone: +8657188122233 \\ Fax: +8657188122233 \\ E-mail: wangkejingmkp@ \\ 163.com
}


or translation inhibition [11]. Emerging evidence indicates that miRNAs play important roles in various key biological processes, including cell proliferation, apoptosis and differentiation [12]. Moreover, dysregulated miRNAs are frequently observed in various tumors, such as miR-34a in breast cancer [13], miR-137 in ovarian cancer [14], miRNA-199a/195 in liver cancer [15] and so on. Depending on the targets of miRNAs, miRNAs may act as oncogenes or tumor suppressors [16]. To date, more and more studies report that miR-345 is downregulated and plays suppressive roles in various types of human cancers. For instance, Chen et al. found that nonsmall cell lung cancer (NSCLC) patients with low miR-345 expression had obviously shorter 5-year overall survival time than those with high miR-345 expression [17]. Chen et al. showed that miR-345 had suppressive activities on prostate cancer proliferation, invasion and migration [18]. Yu et al. proved that miR-345 inhibited the epithelial-mesenchymal transition (EMT) process in hepatocellular carcinoma (HCC) cells and highlighted the potential effects of miR-345 on HCC therapy [19].

However, the roles of miR-345-5p in human PTC pathogenesis are still largely unknown. Here, we investigated the biological effects of miR-345$5 p$ on the proliferation and apoptosis of TPC 1 and B-CPAP cells. Moreover, we confirmed that SETD7 was a direct target of miR-345-5p in PTC cells and found a negative correlation between miR-345-5p and SETD7 expression in PTC tissue and cell lines. In addition, we investigated the possible mechanisms of miR-345-5P induction of the anti-tumor effects on PTC.

\section{Material and methods}

\section{Clinical samples}

Human PTC tissues and neighboring normal tissues were harvested from 15 PTC patients at the time of operation in Zhejiang Cancer Hospital. The samples were quickly snap-frozen in liquid nitrogen and kept at $-70^{\circ} \mathrm{C}$ before use.

\section{Cell lines and cell culture}

Two PTC cell lines (TPC1 and B-CPAP) were purchased from the Type Culture Collection of CAS. Cells were cultured in DMEM medium containing 10\% fetal bovine serum (Gibco, Gaithersburg, MD, USA), $100 \mathrm{U} / \mathrm{ml}$ penicillin (Gibco, Gaithersburg, MD, USA) and $1 \mu \mathrm{g} / \mathrm{ml}$ streptomycin (Gibco, Gaithersburg, MD, USA) at $37^{\circ} \mathrm{C}$ in a humidified atmosphere with $5 \% \mathrm{CO}_{2}$.

\section{MiRNAs and transfection}

MiR-345-5p mimics, scrambled miRNA negative control (NC), anti-miR-345-5p, anti-miRNA negative control (Control), SETD7-shRNA1, SETD7-shRNA2 and scrambled shRNA negative control (Scramble) were designed and synthesized by GenePharma. Cell transfection was conducted using Lipofectamine 2000 (Thermo Scientific, Shanghai, China) follow the manufacturer's instructions. Forty-eight hours after transfection, cells were harvested and used for further analysis.

\section{CCK-8 assay}

Cells (3,000 cells per well) were seeded into 24well plates in normal growth media. After incubation for overnight, cell transfection was conducted and CCK-8 assay was performed at the indicated time points. In brief, $10 \mu \mathrm{l}$ of the CCK-8 solution (Dojindo, Shanghai, China) was added into each well and incubated for $2 \mathrm{~h}$ at $37^{\circ} \mathrm{C}$. Subsequently, the absorbance at $550 \mathrm{~nm}$ was detected via the SpectraMax M5 microplate reader (Molecular devices, Beijing, China).

\section{Flow cytometry analysis}

Flow cytometry analysis was conducted as previously described [20]. At indicated time points after transfection, PTC cells were harvested and incubated with binding buffer containing Annexin V/PI (BD Biosciences) in the dark for $10 \mathrm{~min}$ at room temperature. Then, cells were washed and resuspended in binding buffer. Apoptotic cells were detected by a FACScan flow cytometer (BD Biosciences).

\section{Soft agar colony formation assay}

One thousand cells were trypsinized and seeded into 12-well plates. After 18 days, cells were fixed with formaldehyde $(1 \mathrm{ml} /$ well) for $10 \mathrm{~min}$. After washing, cells were stained with crystal violet $(1 \mathrm{ml} /$ well) for $30 \mathrm{~min}$ and the number of colonies was counted.

\section{Western blot}

Western blot was performed as previously described [21]. Briefly, cells or frozen tissues ground to powder in liquid nitrogen were lysed in RIPA buffer supplemented with $1 \mathrm{mM}$ PMSF. Protein concentration was determined using the BCA protein assay kit (Thermo Scientific, Shanghai, China). Equal amounts of protein $(50 \mu \mathrm{g})$ were separated by SDS-PAGE and transferred onto PVDF membranes (Millipore). After blocking with fat-free dry milk (5\% in PBS), membranes were incubated with rabbit anti-SETD7 (1: 1000, Abcam, Shanghai, China) and rabbit anti-GADPH (1:500, Abcam, Shanghai, China) overnight at $4^{\circ} \mathrm{C}$. Following extensive washes, the signal on the blot was examined with HRP-conjugated secondary antibodies and ECL (Millipore, Shanghai, China). 


\section{Luciferase reporter assay}

SETD7 3'-UTR, harboring the putative miR-345$5 p$ binding sites, was synthesized and cloned into pGL4 (Promega, Madison, WI, USA) to generate wild-type plasmid (SETD7-WT). The mutant SETD7 3'-UTR, harboring the mutant putative binding sequences of miR-345-5p, was cloned into pGL4 to generate the mutant report plasmid (SETD7MT). TPC1 and B-CPAP cells were transfected with miR-345-5p mimics or miR-NC, and SETD7-WT or SETD7-MT. At $48 \mathrm{~h}$, a luciferase reporter assay was conducted via the dual luciferase assay system (Promega, Madison, WI, USA).

\section{Quantitative real-time PCR (qPCR) analysis}

qPCR analysis was carried out as previously described [22]. In brief, total RNA was prepared from cells or tissues using TRIzol Reagent (Takara, Tokyo, Japan) and reverse transcription was carried out using a cDNA Reverse Transcription kit (Takara, Tokyo, Japan). Bestar Real time PCR Master Mix (TaKaRa, Tokyo, Japan) was employed to examine miR-345-5p and SETD7 expression levels. All PCR reactions were performed via the $A B I 7500$ PCR System (Applied Biosystems, Carlsbad, California, USA). Relative expression levels of above genes were calculated by the $2^{-\Delta \Delta C t}$ method and GAPDH was used as an input control. The primers used for qPCR were:

SETD7-fwd: 5'-CCTGGTGGAAGTTAGGTGCTA-3';

SETD7-rev: 5'-CGGTGTCTCTAATGCCTCTGA-3'; miR-345-5p-fwd: 5'-TCGGCGGCTGACTCCTAGTCCA-3';

miR-345-5p- rev: 5'-GTCGTATCCAGTGCAGGGTCCGAGGT-3';

GAPDH-fwd: 5'-TGCACCACCAACTGCTTAGC-3'; GAPDH-rev: 5'-GGCATGGACTGTGGTCATGAG-3'.

\section{Statistical analysis}

At least three independent tests were performed and results were presented as mean $\pm S D$. All the data were analyzed by one-way ANOVA, and $p \leq 0.05$ was considered to indicate statistical significance.

\section{Results}

\section{There was low miR-345-5p expression} and high SETD7 expression in PTC

Previous studies have shown that downregulation of miR-345 is often observed in different tumors. In this study, qPCR was employed to analyze the mRNA levels of miR-345-5p and SETD7 and the results showed that there was a lower miR-345-5p mRNA level (Figure $1 \mathrm{~A}, p<0.001$ ) and a higher SETD7 mRNA level (Figure $1 \mathrm{~B}, p<$ 0.001 ) in PTC tissues, compared with neighbor- ing normal tissues. Figure $1 \mathrm{C}$ also indicated that SETD7 protein level was increased in PTC tissues compared with neighboring normal tissues $(p<$ 0.001). Moreover, Spearman's correlation analysis demonstrated that there was a negative correlation between miR-345-5p and SETD7 expression in PTC tissues (Figure $1 \mathrm{D}$ ), which was consistent with the above data.

\section{MiR-345-5p overexpression inhibited proliferation and promoted apoptosis of PTC cells}

To test the effect of miR-345-5p on the cell proliferation and apoptosis in PTC cells, TPC1 and B-CPAP cells were transfected with miR-345-5p mimics or anti-miR-345-5p. Figure 2 A showed that the miR-345-5p level was increased in TPC1 and B-CPAP cells transfected with miR-345-5p mimics $(p<0.001)$, whereas the expression of miR-345-5p was significantly decreased in the anti-miR-345-5p group, compared to matched control groups $(p<0.01)$. CCK-8 assay revealed that from the second day after transfection, antimiR-345-5p significantly promoted the viability of TPC1 and B-CPAP cells, whereas miR-345-5p mimics significantly inhibited the growth of PTC cells, compared to matched control groups (Figure $2 \mathrm{~B}$, $p<0.05$ for day 2 ). In addition, Figure 2 C shows that miR-345-5 mimics inhibited colony formation of PTC cells $(p<0.001)$, whereas anti-miR$345-5 p$ promoted colony formation of PTC cells ( $p<0.01$ and $p<0.001$ for TPC1 and B-CPAP cells, respectively). Moreover, Figure $2 \mathrm{D}$ demonstrates that miR-345-5p mimics increased the apoptosis of TPC1 and B-CPAP cells compared with NC ( $p<0.001$ and $p<0.01$ for TPC1 and B-CPAP cells, respectively), whereas anti-miR-345-5p inhibited apoptosis $(p<0.01$ and $p<0.001$ for TPC1 and $\mathrm{B}$-CPAP cells, respectively).

\section{SETD7 was a direct target of miR-345-5p in PTC cells}

Target scan analysis showed that SETD7 was the potential target of miR-345-5p. Figure $3 \mathrm{~A}$ showed that SETD7 3'-UTR had the complementary site for the miR-345-5p seed region. Hence, we constructed reporter vectors containing the luciferase coding sequence followed by the wildtype or mutant of SETD73'-UTR. Figure 3 B showed that miR-345-5p mimics suppressed the luciferase activity of SETD7-WT compared with miR-NC $(p<0.01)$, whereas it had no significant effect on the luciferase activity of SETD7-MT, which indicated the interruption of the interaction of miR-345$5 p$ with SETD7. Figure $3 \mathrm{C}$ shows that TPC1 and B-CPAP cells transfected with miR-345-5p mimics had lower SETD7 mRNA and protein levels com- 
A

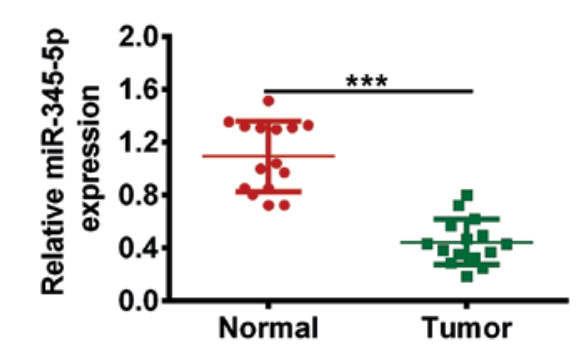

B

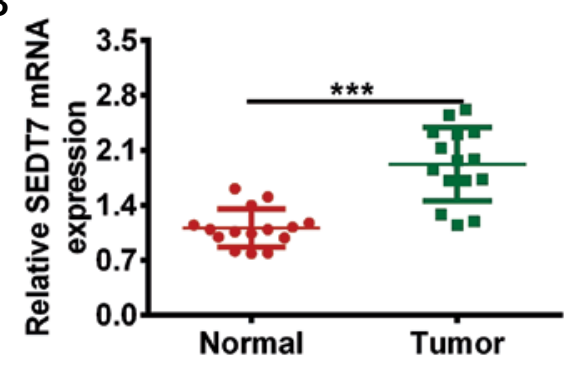

C
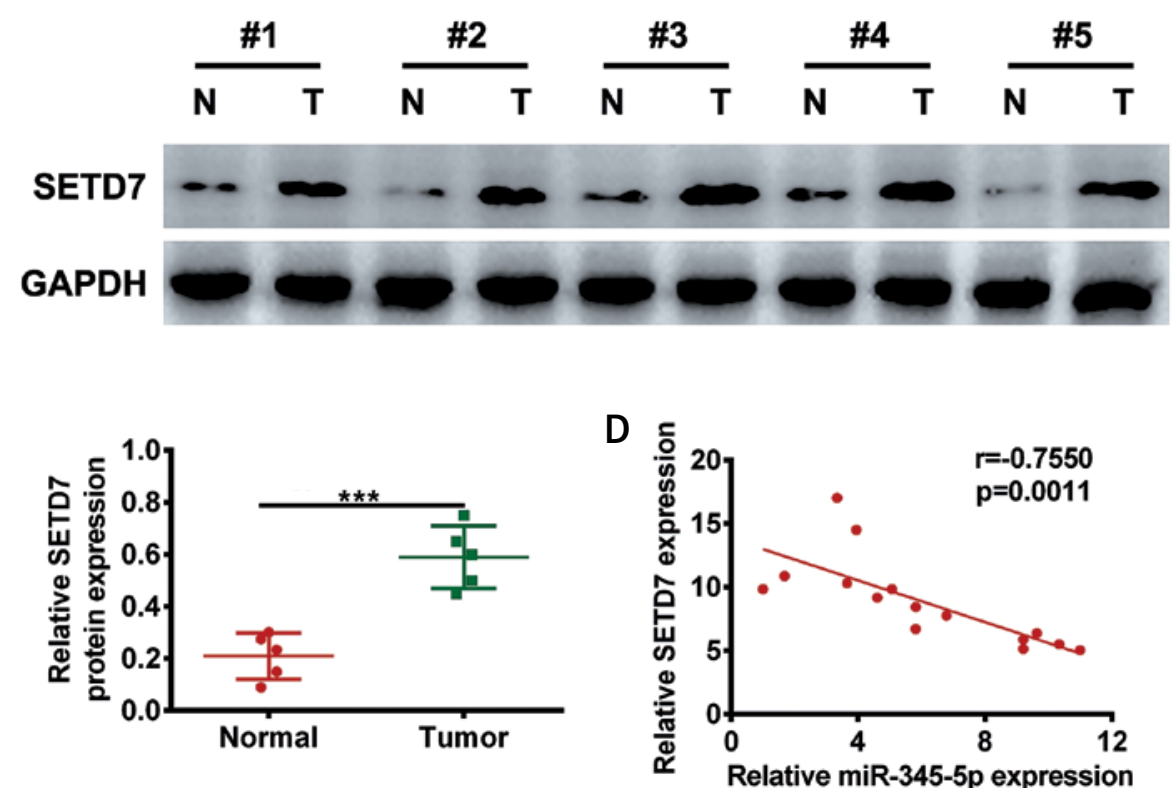

Figure 1. Expression levels of miR-345-5p and SETD7 in PTC samples and neighboring normal tissues. A, B - MiR345-5p (A) or SETD7 (B) expression level in PTC samples (Tumor) and neighboring normal tissues (Normal) was determined by qPCR. C - SETD7 protein level in PTC samples (T) and neighboring normal tissues (N) collected from 15 patients was analyzed through Western blot. $\mathbf{D}$ - The relationship between miR-345-5p and SETD7 mRNA level in PTC tissues was determined via qPCR and Spearman's correlation analysis

${ }^{* * *} p<0.001$.

pared to cells in NC groups $(p<0.001)$. In contrast, knockdown of miR-345-5p increased SETD7 mRNA $(p<0.01)$ and protein level $(p<0.001)$ in PTC cells, compared with the control (Figure $3 \mathrm{D}$ ). Thus, the above data showed that SETD7 was a direct target of miR-345-5p in PTC cells.

\section{SETD7 promoted PTC cell proliferation and inhibited apoptosis}

To study the functional effects of SETD7 on PTC development, SETD7 cDNA was amplified and ligated into pCDNA3.1 vector to construct the SETD7 overexpression plasmid. TPC1 and B-CPAP cells were transfected with SETD7 overexpression plasmid or SETD7 shRNAs. QPCR and Western blot data showed that PTC cells transfected with SETD7 overexpression plasmid had higher SETD7 mRNA (Figure $4 \mathrm{~A}, p<0.001$ ) and protein levels (Figure $4 \mathrm{~B}, p<0.01$ ), compared to NC groups. In contrast, downregulation of SETD7 with transfection of shRNAs significantly decreased SETD7 mRNA
(Figure $4 \mathrm{~A}, p<0.001$ ) and protein levels (Figure $4 \mathrm{~B}, p<0.001$ and $p<0.01$ for TPC1 and B-CPAP cells, respectively) in PTC cells compared with the control. Figure $4 \mathrm{C}$ demonstrated that overexpression of SETD7 promoted the viability of PTC cells compared to control cells ( $p<0.001$ for day 5 ) in a time-dependent manner, whereas downregulation of SETD7 inhibited the above phenomenon $(p<0.001$ for day 5$)$. In addition, Figure $4 \mathrm{D}$ shows that overexpression of SETD7 enhanced the colony formation of PTC cells $(p<0.01$ and $p<0.001$ for TPC1 and B-CPAP cells, respectively), whereas downregulation of SETD7 by shRNA1 $(p<0.01$ and $p<0.05$ for TPC1 and B-CPAP cells, respectively) and SETD7 shRNA2 $(p<0.001$ and $p<0.01$ for TPC1 and B-CPAP cells, respectively) inhibited the colony formation of tumor cells. Furthermore, Figure $4 \mathrm{E}$ shows that overexpression of SETD7 inhibited the apoptosis of PTC cells $(p<0.001)$, whereas downregulation of SETD7 promoted cell apoptosis $(p<0.001$ and $p<0.01$ for TPC1 and B-CPAP cells, respectively). 
A
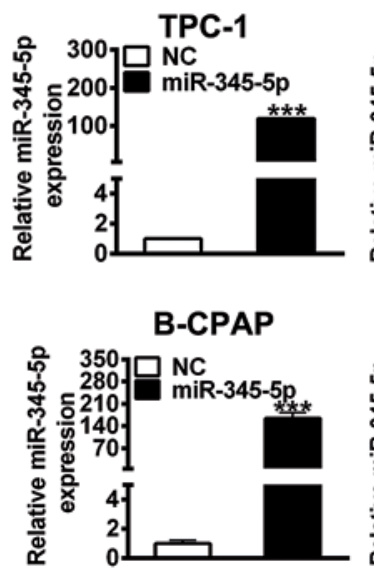

C

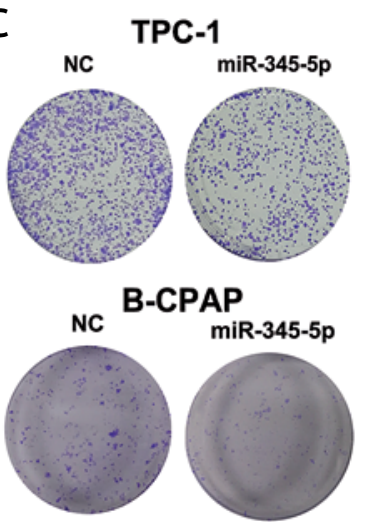

D

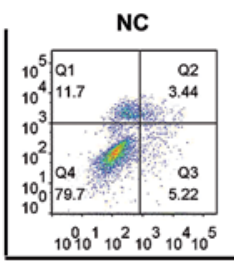

miR-345-5p
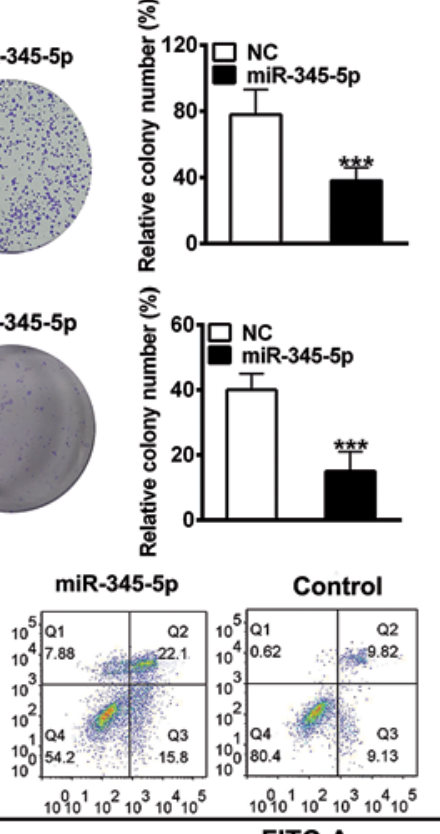

FITC-A
TPC-1

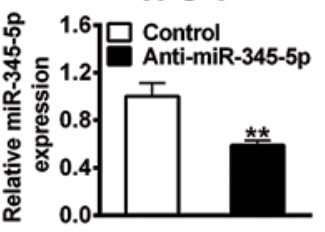

B-CPAP
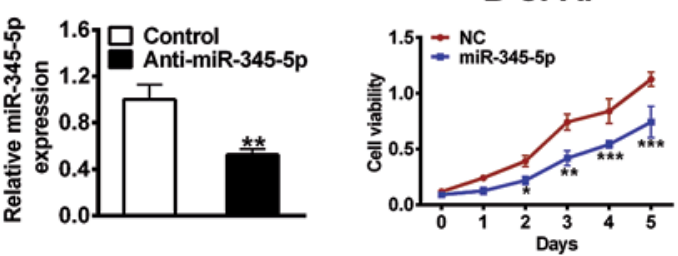

TPC-1

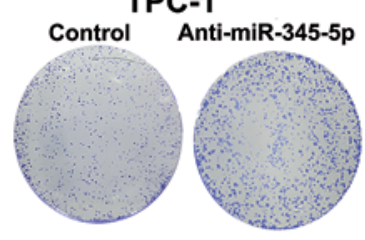

B-CPAP

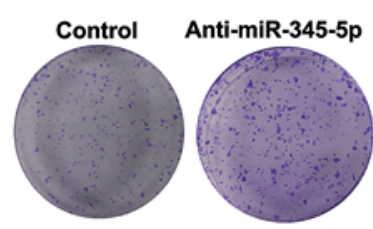

Anit-miR-345-5p
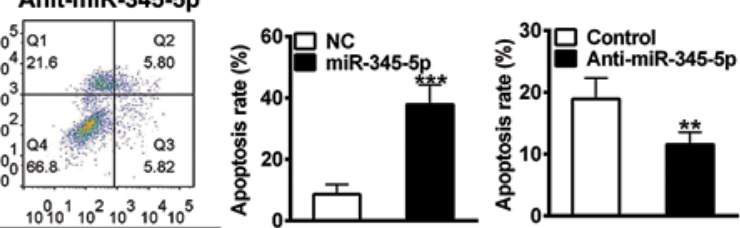

TPC-1

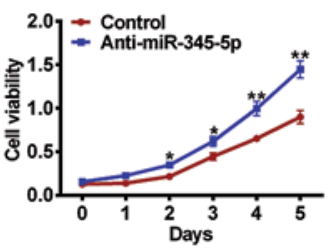

B-CPAP
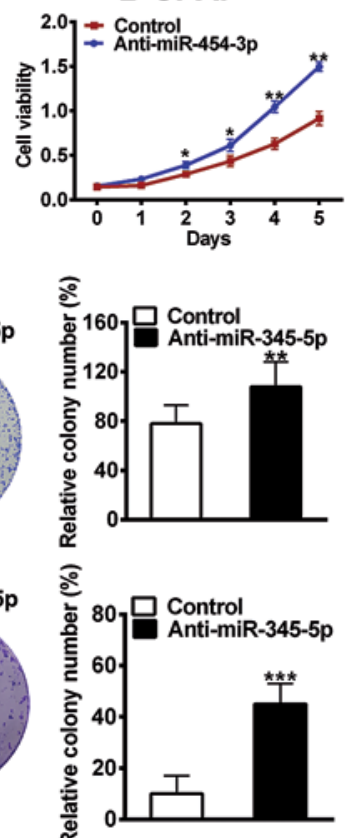
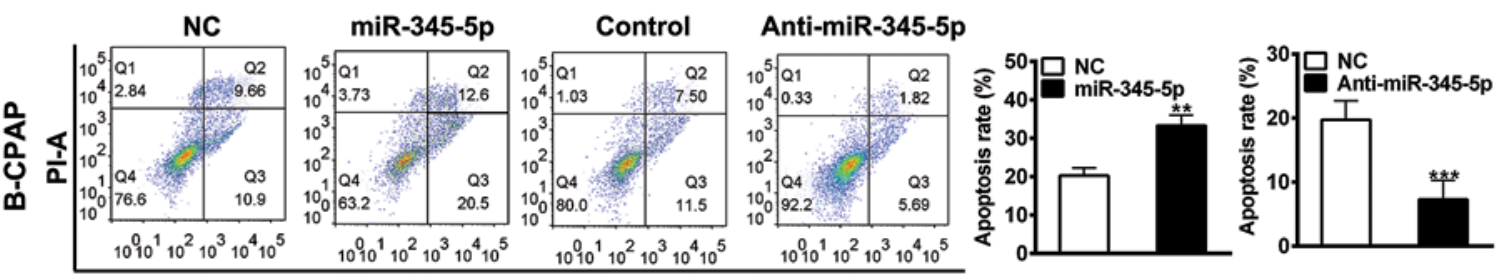

Figure 2. Effect of miR-345-5p on the proliferation and apoptosis of PTC cells. TPC1 and B-CPAP cells were transfected with miR-345-5p mimics or anti-miR-345-5p (A) MiR-345-5p levels were analyzed by qPCR (B, C). CCK-8 (B) and soft agar colony formation assay of transfected PTC cells (C). D - Apoptosis of transfected PTC cells was detected by Annexin $\mathrm{V}$ and PI double staining followed by analysis with flow cytometry

$$
{ }^{\star} p<0.05 ;{ }^{* *} p<0.01 ;{ }^{* * *} p<0.001 \text {. }
$$

SETD7 overexpression blocked the anti-tumor effects of miR-345-5p on PTC cells

Figure $5 \mathrm{~A}$ confirmed that miR-345-5p mimics inhibited the viability of TPC1 and B-CPAP cells compared to the negative control (NC), whereas SETD7 overexpression plasmid abolished the above inhibition effect by miR-345-5p mimics $(p<0.001$ for day 5$)$. Figure $5 \mathrm{~B}$ revealed that miR$345-5 p$ mimics led to a significant decrease in the colony number of PTC cells $(p<0.001)$, whereas the colony number increased after the co-transfection with SETD7 overexpression plasmid and miR-345-5p mimics $(p<0.001$ and $p<0.05$ for 
A

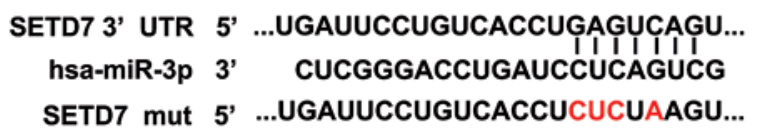

C

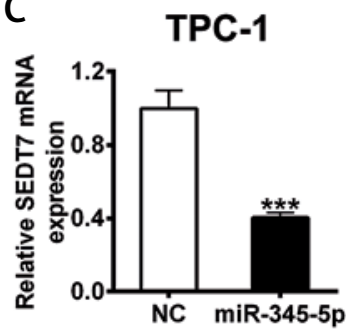

TPC-1
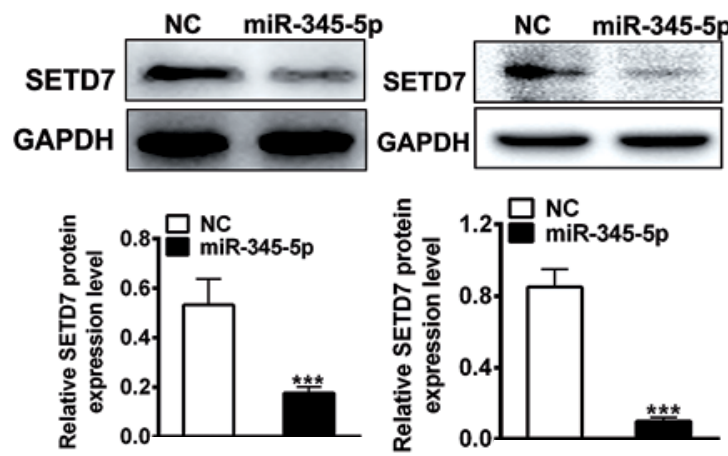

B

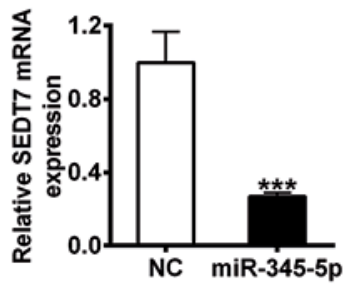

B-CPAP

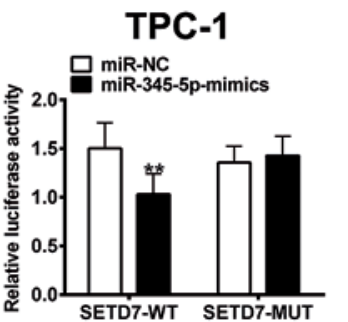

TPC-1

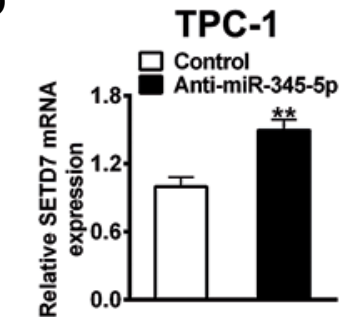

TPC-1

Anti-
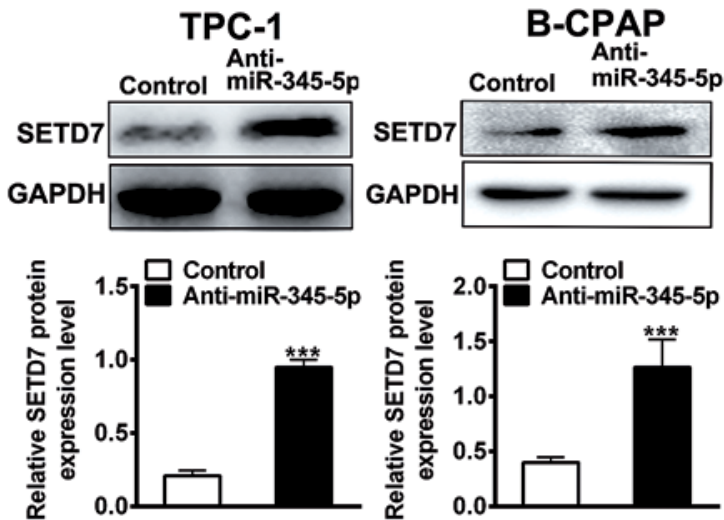

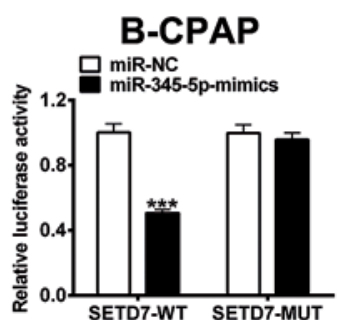

B-CPAP
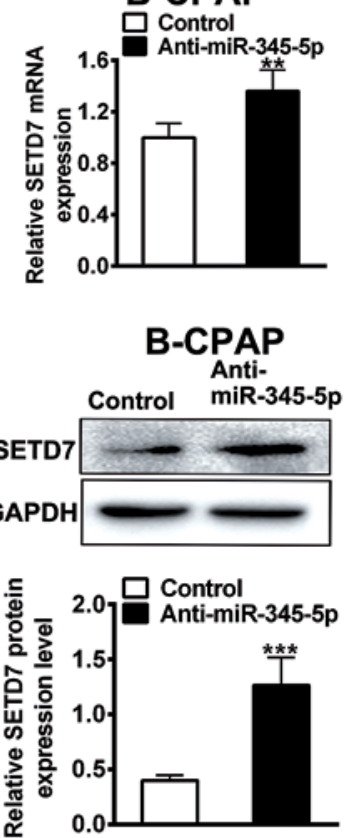

Figure 3. Identification the target of miR-345-5p in TPC1 and B-CPAP cells. A - Schematic diagram of miR-345$5 p$ target sites within SETD7 3'-UTR. Sequences were compared between the mature miR-345-5p and wild-type or mutant putative binding sites in SETD73'-UTR. B - Luciferase reporter activities driven by wild-type or mutant SETD7 3'-UTR were examined in PTC cells transfected with miR-345-5p mimics. C - MiR-345-5p and SETD7 mRNA levels and SETD7 protein level in PTC cells transfected with miR-345-5p mimics were determined by qPCR and Western blot, respectively. D - MiR-345-5p expression level, SETD7 mRNA and protein levels in PTC cells transfected with anti-miR-345-5p were determined by qPCR and Western blot, respectively

${ }^{* *} p<0.01 ;{ }^{* * *} p<0.001$.

TPC1 and B-CPAP cells, respectively). In addition, Figure $5 \mathrm{C}$ indicates that the apoptosis rates of cells in miR-34a mimic groups was increased compared to those in the negative control (NC) groups $(p<0.001)$, whereas SETD7 overexpression plasmid significantly inhibited miR-34a mimic induced apoptosis $(p<0.01)$.

\section{Discussion}

The dysregulation of miRNAs often occurs in various human tumors and plays vital roles in regulating the expression of multiple target genes [5]. Recently, a number of miRNAs were reported to be dysregulated in PTC and were closely related to the initiation and progression of PTC. Wang et al. reported that miR-663 played tumor suppressive roles in EMT progress of PTC cells [23]. Ye et al. revealed that miR-139 exerted a suppressive effect on PTC tumorigenesis through targeting FN1 [24].
Huang et al. found that miR-219-5p negatively regulated PTC growth by directly targeting and inhibiting ER $\alpha$. Here, we found that miR-345-5p expression level was decreased in PTC tissues. Moreover, miR-345-5p overexpression inhibited the viability and colony formation, and promoted apoptosis of TPC1 and B-CPAP cells. In contrast, downregulation of miR-345-5p significantly induced PTC cell proliferation and suppressed apoptosis. Thus, miR-345-5p may act as a tumor suppressor in PTC cells.

MiRNAs could function as oncogenes or tumor suppressors via controlling expression of their targets related to tumorigenesis or tumor progression [25]. To date, two novel targets of miR-345, i.e. IRF1 in hepatocellular carcinoma (HCC) [19] and Smad1 in human prostate cancer [18], have been confirmed. In our study, target scan analysis suggested that SETD7 was a potential target of miR-345-5p. Moreover, luciferase reporter assay 
A
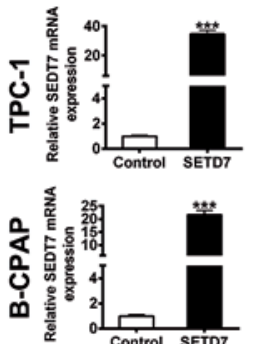

$\mathrm{C}$
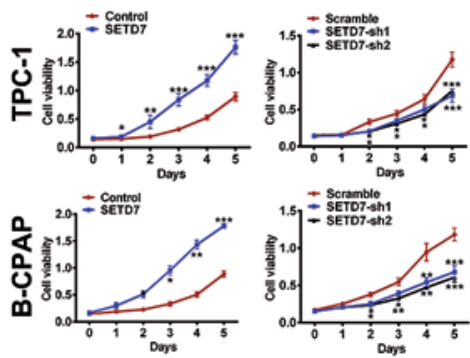
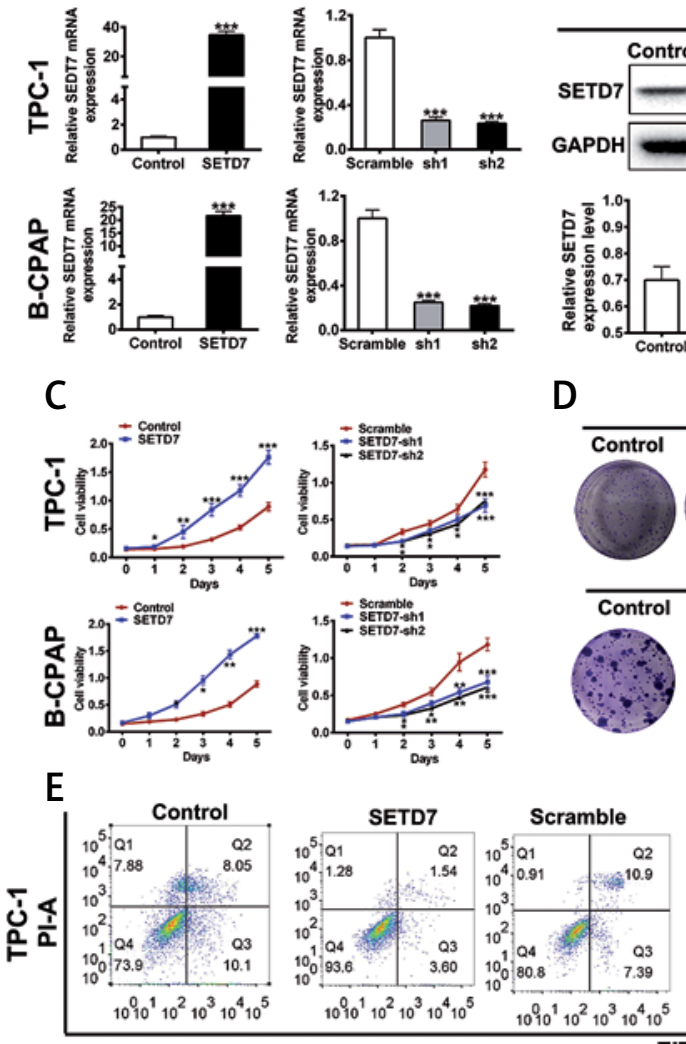

TPC-1

B
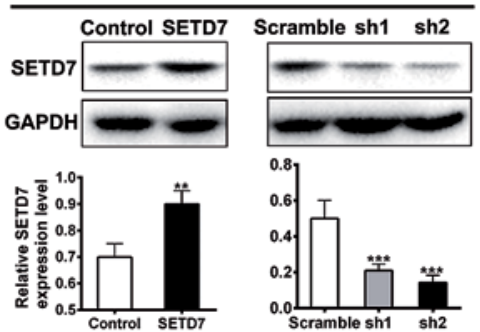

D

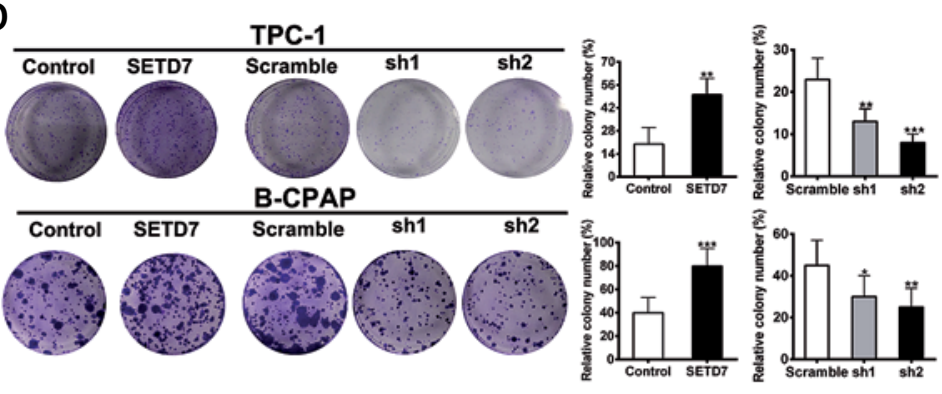

B-CPAP
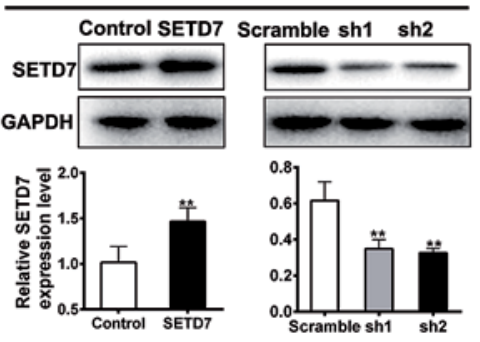
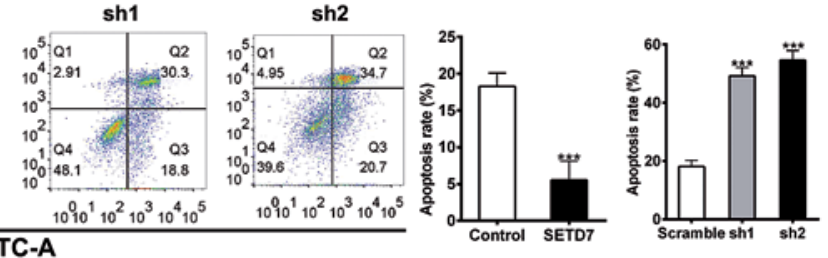

\section{FITC-A}

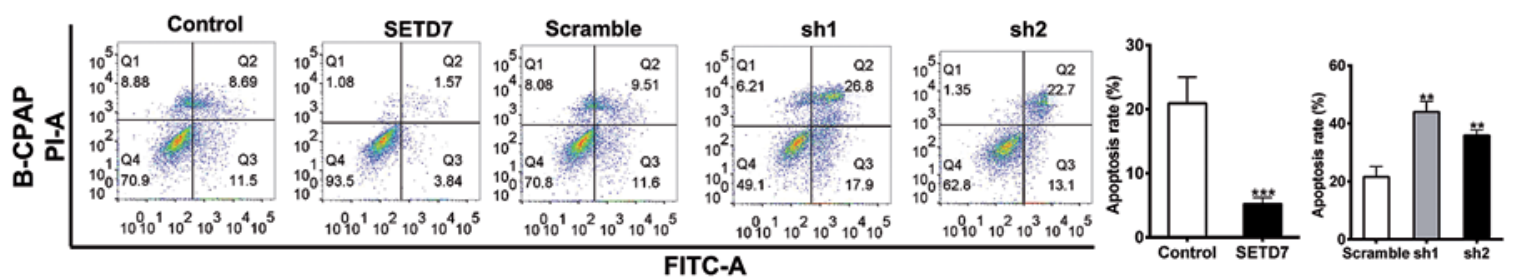

Figure 4. Effect of SETD7 on proliferation and apoptosis of PTC cells. TPC1 and B-CPAP cells were transfected with SETD7 overexpression plasmid or SETD7 shRNA1 or SETD7 shRNA2. A, B - SETD7 mRNA (A) and protein (B) levels were analyzed by qPCR and Western blot, respectively. C, D - CCK-8 (C) and soft agar colony formation assay (D) of transfected cells. $\mathbf{E}-$ Apoptosis of transfected cells was detected

${ }^{*} p<0.05 ;{ }^{* *} p<0.01 ;{ }^{* * *} p<0.001$.

further identified that miR-345-5p overexpression significantly inhibited the luciferase activity of wild-type SETD7 3'-UTR. Additionally, we found that miR-345-5p mimics decreased SETD7 mRNA and protein levels in TPC1 and B-CPAP cells, and conversely, downregulation of miR-345-5p increased SETD7 mRNA and protein levels in PTC cells. Therefore, these data indicated that SETD7 was a direct target of miR-345-5p in PTC cells.

Epigenetic changes play vital functions in the onset and progression of various cancers. Methylation is one type of epigenetic modifications and has been intensively probed [26]. SETD7, also known as SET7, SET9, KMT7 and SET7/9, is one of the SET domain-containing proteins and has been proved to monomethylate $\mathrm{H} 3 \mathrm{~K} 4$ [27] and numerous non-histone proteins, such as p53 [28], DNMT1 [29], YAP [30] and so on. In the last few decades, many studies have indicated that high methylation is one of the major features of tumor cells. Accumulating evidence shows that the aberrant expression of SETD7 is closely related to the progression of cancers. For instance, Chen et al. revealed that SETD7 overexpression induced HCC cell proliferation, whereas SETD7 knockdown suppressed HCC cell proliferation [31]. Huang et al. demonstrated that SETD7 induced breast cancer cell proliferation and suppressed cell apoptosis, which suggested that SETD7 expression level can predict the prognosis of breast cancer [32]. However, Gu et al. reported that SETD7 promoted apoptosis of acute myeloid leukemia cells and prohibited apoptosis of NSCLC cells, indicating opposite effects of SETD7 on tumor cell apoptosis and tumorigenesis depending on different tumor types [33]. 
A

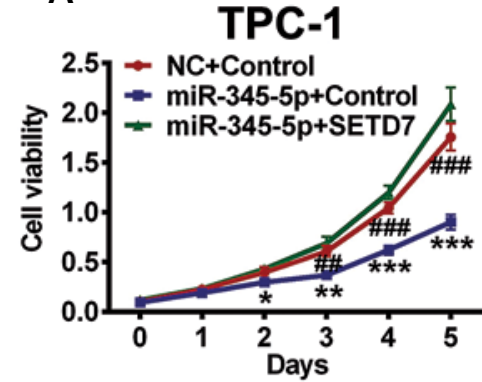

B-CPAP

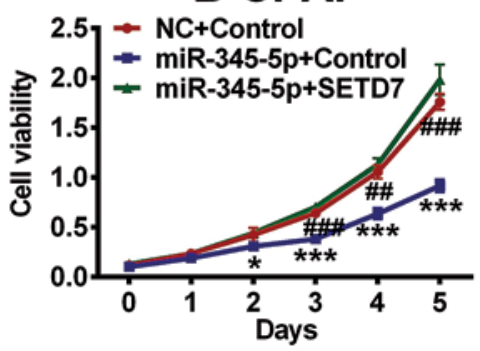

C
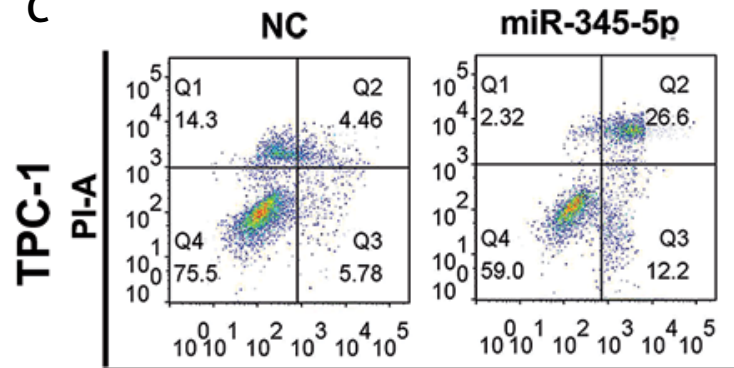

FITC-A
TPC-1

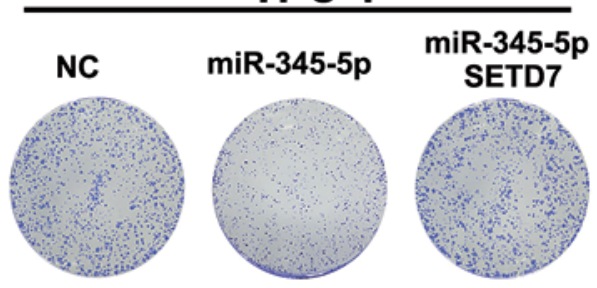

B-CPAP

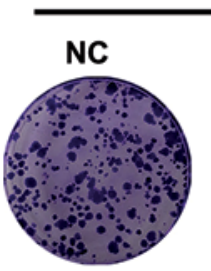
miR-345-5p miR-345-5p +SETD7

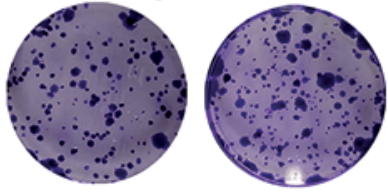

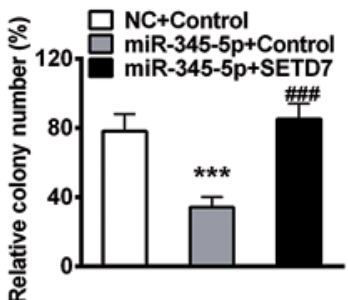
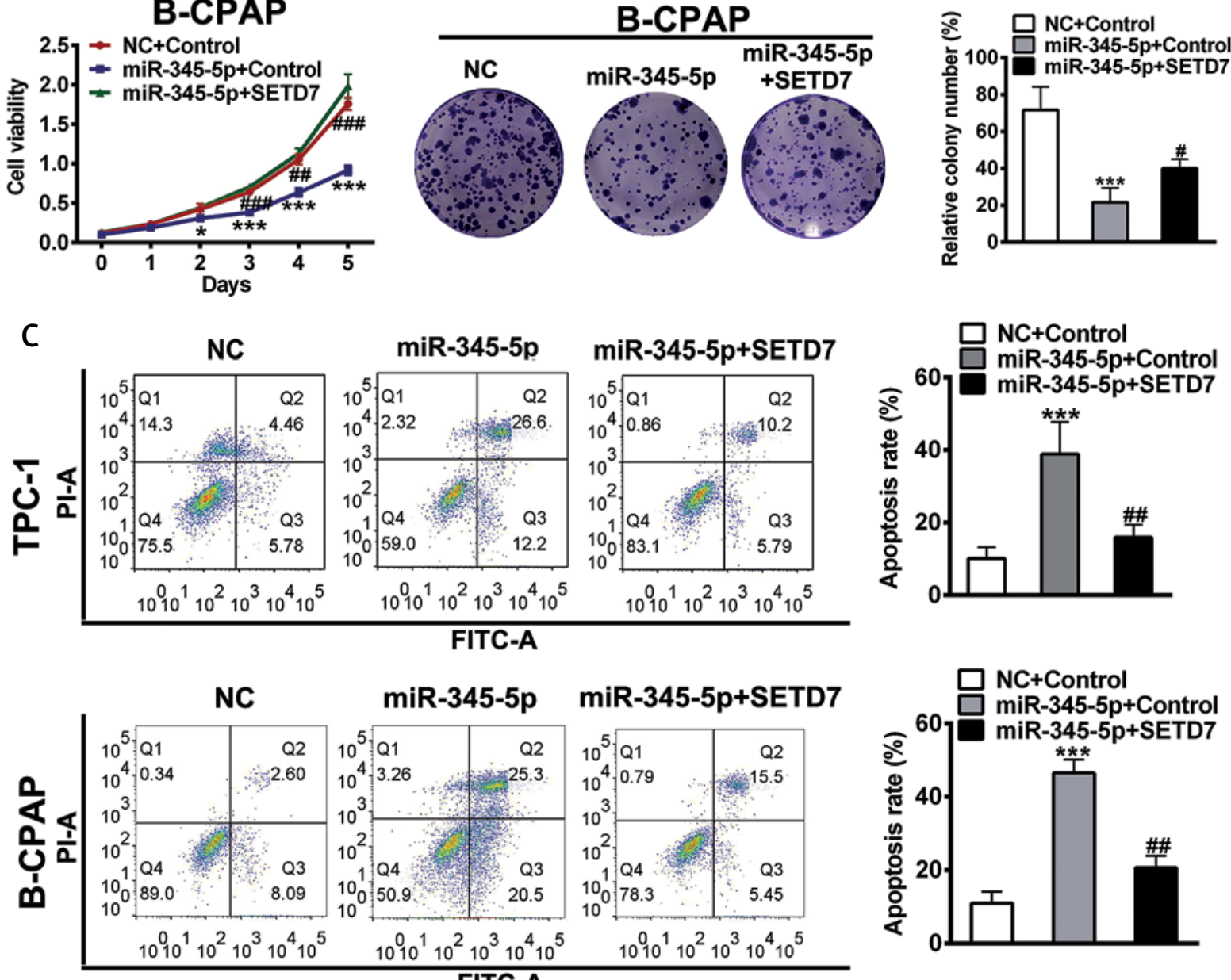

miR-345-5p+SETD7
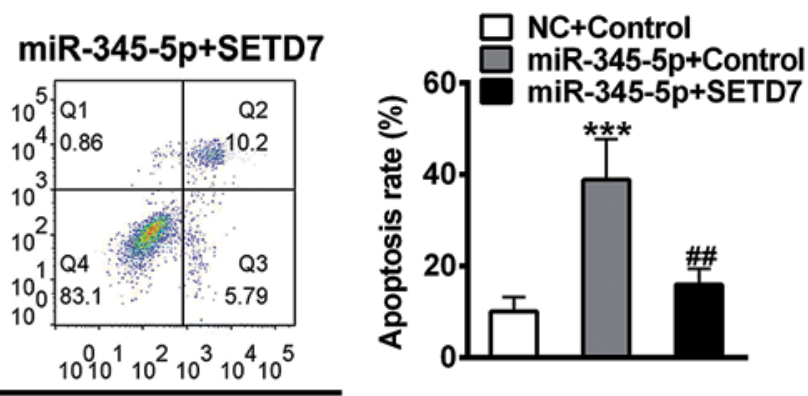

Figure 5. Proliferation, colony formation ability and apoptosis assay of PTC cells transfected with miR-345-5p mimics, as well as empty vector or SETD7 overexpression vector. TPC1 and B-CPAP cells were transfected with miR345-5p mimics, as well as empty vector (Control) or SETD7 overexpression vector. A - CCK-8 assay of PTC cells at indicated time points after transfection. B - Soft agar colony formation assay of PTC cells at day 12. C - Apoptosis of transfected cells was detected

${ }^{*} p<0.05,{ }^{* *} p<0.01$ and ${ }^{* *} p<0.001$ compared to NC group; ${ }^{*} p<0.05,{ }^{* \#} p<0.01$ and ${ }^{\# \# *} p<0.001$ compared to miR-345-5p group.

Here, we found that overexpression of SETD7 promoted PTC cell proliferation and inhibited apoptosis, whereas the downregulation of SETD7 had opposite effects on PTC cells. Moreover, SETD7 overexpression abolished the miR-345-5p induced anti-tumor effects in PTC cells. On the other hand, Western blot results showed that PTC tissues of patients had a significantly higher level of SETD7 than that of the neighboring normal tissues. Fur- thermore, there was a negative correlation between miR-345-5p and SETD7 expression in PTC tissues. Taken together, our results indicated that the tumor suppressive roles of miR-345-5p on PTC may be a result of its negative regulation of SETD7 expression. However, further studies are still needed to identify the downstream targets of SETD7 in PTC.

In conclusion, the above data revealed that low miR-345-5p expression and high SETD7 expression 
in PTC cells were closely related to the progression of PTC, and miR-345-5p acted as a tumor repressor through negatively regulating of the expression of SETD7 in PTC cells. Thus, our findings suggested that miR-345-5p could be a potential diagnostic factor and therapeutic target for PTC. MiR-345-5p replacement therapy inhibited the oncogenic roles of SETD7, which subsequently inhibited PTC cell proliferation and induced apoptosis. Hence, the present study also encouraged a rational application of SETD7 inhibitor in PTC therapy. However, the in vivo anti-tumor roles of miR-345-5p or SETD7 inhibitor warrant further investigation.

MiR-345-5 $p$ acted as a tumor repressor through targeting SETD7, and subsequently played an important role in controlling PTC cell proliferation and apoptosis.

\section{Acknowledgments}

This study was supported by Zhejiang Cancer Hospital. Our study was approved by the Institutional Ethics Committee of Zhejiang Cancer Hospital, and conducted in accordance with the Helsinki Declaration. Written informed consent was collected from all patients prior to surgery.

\section{Conflict of interest}

The authors declare no conflict of interest.

\section{References}

1. Sipos JA, Mazzaferri EL. Thyroid cancer epidemiology and prognostic variables. Clin Oncol (R Coll Radiol) 2010; 22: 395-404.

2. Loh KC, Greenspan FS, Gee L, Miller TR, Yeo PP. Pathological tumor-node-metastasis (pTNM) staging for papillary and follicular thyroid carcinomas: a retrospective analysis of 700 patients. J Clin Endocrinol Metab 1997; 82: 3553-62.

3. Qiu Z, Li H, Wang J, Sun C. miR-146a and miR-146b in the diagnosis and prognosis of papillary thyroid carcinoma. Oncol Rep 2017; 38: 2735-40.

4. Brown RL, de Souza JA, Cohen EE. Thyroid cancer: burden of illness and management of disease. J Cancer 2011; 2: 193-9.

5. Bartel DP. MicroRNAs: genomics, biogenesis, mechanism, and function. Cell 2004; 116: 281-97.

6. Lima CR, Geraldo MV, Fuziwara CS, Kimura ET, Santos MF. MiRNA-146b-5p upregulates migration and invasion of different papillary thyroid carcinoma cells. BMC Cancer 2016; 16: 108

7. Nikiforova MN, Nikiforov YE. Molecular genetics of thyroid cancer: implications for diagnosis, treatment and prognosis. Expert Rev Mol Diagn 2008; 8: 83-95.

8. Lang BH, Wong KP, Wan KY, Lo CY. Significance of metastatic lymph node ratio on stimulated thyroglobulin levels in papillary thyroid carcinoma after prophylactic unilateral central neck dissection. Ann Surg Oncol 2012; 19: 1257-63.

9. Bhardwaj A, Singh S, Singh AP. MicroRNA-based cancer therapeutics: big hope from small RNAs. Mol Cell Pharmacol 2010; 2: 213-9.
10. Yang M, Zhang L, Wang X, Zhou Y, Wu S. Down-regulation of miR-203a by IncRNA PVT1 in multiple myeloma promotes cell proliferation. Arch Med Sci 2018; 14: 1333-9.

11. He H, Jazdzewski K, Li W, et al. The role of microRNA genes in papillary thyroid carcinoma. Proc Natl Acad Sci USA 2005; 102: 19075-80.

12. Tang JT, Wang JL, Du W, et al. MicroRNA 345, a methylation-sensitive microRNA is involved in cell proliferation and invasion in human colorectal cancer. Carcinogenesis 2011; 32: 1207-15.

13. Wu MY, Fu J, Xiao X, Wu J, Wu RC. MiR-34a regulates therapy resistance by targeting HDAC1 and HDAC7 in breast cancer. Cancer Lett 2014; 354: 311-9.

14. Dong P, Xiong Y, Watari H, et al. MiR-137 and miR-34a directly target Snail and inhibit EMT, invasion and sphere-forming ability of ovarian cancer cells. J Exp Clin Cancer Res 2016; 35: 132.

15. Li Y, Di C, Li W, et al. Oncomirs miRNA-221/222 and tumor suppressors miRNA-199a/195 are crucial miRNAs in liver cancer: a systematic analysis. Dig Dis Sci 2016; 61: 2315-27.

16. Srivastava SK, Bhardwaj A, Arora S, et al. MicroRNA-345 induces apoptosis in pancreatic cancer cells through potentiation of caspase-dependent and -independent pathways. Br J Cancer 2015; 113: 660-8.

17. Chen L, Li X, Chen X. Prognostic significance of tissue miR-345 downregulation in non-small cell lung cancer. Int J Clin Exp Med 2015; 8: 20971-6.

18. Chen QG, Zhou W, Han T, et al. MiR-345 suppresses proliferation, migration and invasion by targeting Smad1 in human prostate cancer. J Cancer Res Clin Oncol 2016; 142: 213-24.

19. Yu $M$, Xue $H$, Wang $Y$, et al. miR-345 inhibits tumor metastasis and EMT by targeting IRF1-mediated mTOR/ STAT3/AKT pathway in hepatocellular carcinoma. Int J Oncol 2017; 50: 975-83.

20. Pandurangan AK, Ismail S, Esa NM, Munusamy MA. Inositol-6 phosphate inhibits the mTOR pathway and induces autophagy-mediated death in HT-29 colon cancer cells. Arch Med Sci 2018; 14: 1281-8.

21. Li X, Li M, Chen D, Shi G, Zhao H. PAQR3 inhibits proliferation via suppressing PI3K/AKT signaling pathway in non-small cell lung cancer. Arch Med Sci 2017; 14: 1289-97.

22. Luo Z, Pan J, Ding Y, Zhang YS, Zeng Y. The function and clinical relevance of IncRNA UBE2CP3-001 in human gliomas. Arch Med Sci 2018; 14: 1308-20.

23. Wang Z, Zhang H, Zhang P, Dong W, He L. MicroRNA-663 suppresses cell invasion and migration by targeting transforming growth factor beta 1 in papillary thyroid carcinoma. Tumour Biol 2016; 37: 7633-44.

24. Ye Y, Zhuang J, Wang G, He S, Ni J, Xia W. MicroRNA-139 targets fibronectin 1 to inhibit papillary thyroid carcinoma progression. Oncol Lett 2017; 14: 7799-806.

25. Minna E, Romeo P, De Cecco L, et al. miR-199a-3p displays tumor suppressor functions in papillary thyroid carcinoma. Oncotarget 2014; 5: 2513-28.

26. Ropero S, Esteller M. The role of histone deacetylases (HDACs) in human cancer. Mol Oncol 2007; 1: 19-25.

27. Benevolenskaya EV. Histone H3K4 demethylases are essential in development and differentiation. Biochem Cell Biol 2007; 85: 435-43.

28. Chuikov S, Kurash JK, Wilson JR, et al. Regulation of p53 activity through lysine methylation. Nature 2004; 432: 353-60.

29. Esteve PO, Chin HG, Benner J, et al. Regulation of DNMT1 stability through SET7-mediated lysine methyl- 
ation in mammalian cells. Proc Natl Acad Sci USA 2009;

106: 5076-81.

30. Oudhoff MJ, Braam MJS, Freeman SA, et al. SETD7 Controls intestinal regeneration and tumorigenesis by regulating Wnt/beta-catenin and hippo/YAP signaling. Dev Cell 2016; 37: 47-57.

31. Chen Y, Yang S, Hu J, Yu C, He M, Cai Z. Increased expression of SETD7 promotes cell proliferation by regulating cell cycle and indicates poor prognosis in hepatocellular carcinoma. PLoS One 2016; 11: e0154939.

32. Huang R, Li X, Yu Y, et al. SETD7 is a prognosis predicting factor of breast cancer and regulates redox homeostasis. Oncotarget 2017; 8: 94080-90.

33. Gu Y, Wang Y, Wang X, Gao L, Yu W, Dong WF. Opposite effects of SET7/9 on apoptosis of human acute myeloid leukemia cells and lung cancer cells. J Cancer 2017; 8: 2069-78. 